\title{
Patterns and predictors of functional recovery from the subacute to the chronic phase following a traumatic spinal cord injury: a prospective study
}

\author{
Andréane Richard-Denis ${ }^{1,2} \cdot$ Rami Chatta $^{1} \cdot$ Cynthia Thompson ${ }^{2} \cdot$ Jean-Marc Mac-Thiong ${ }^{1,2,3,4}$
}

Received: 19 December 2018 / Revised: 6 August 2019 / Accepted: 7 August 2019 / Published online: 28 August 2019

(c) The Author(s), under exclusive licence to International Spinal Cord Society 2019

\begin{abstract}
Study design Prospective cohort study.

Objectives To determine the extent of functional recovery between 6 and 12 months following a traumatic spinal cord injury (TSCI) and to identify individuals achieving a small clinical functional improvement during this period.

Setting A single level-1 trauma center specialized in SCI care.

Methods A cohort of 125 patients sustaining TSCI was studied. The Spinal Cord Independence Measure (SCIM) version III at 6 and 12 months post injury was used as the main outcome measure.

Results The observed functional improvement for the final cohort did not reach a clinically significant level between 6 and 12 months post injury. However, $30.4 \%$ of individuals achieved this level ( $\geq 4$ points in the SCIM-III total score). This group showed a higher proportion of motor-complete TSCI (AIS grade A or B) and showed a tendency toward older age and higher trauma severity. Longer duration of intensive functional rehabilitation was the single factor associated with reaching a small clinically important improvement in the SCIM-III total score.

Conclusions Functional status between 6 and 12 months following a TSCI may be considered clinically similar, regardless of the level of injury. However, $30 \%$ may reach a small clinical functional improvement in the subacute to chronic phase following TSCI, particularly individuals sustaining severe deficits and older age, which may highlight the importance of functional compensation during this period for these patients.
\end{abstract}

\section{Introduction}

Traumatic spinal cord injury (TSCI) has profound effects on patient's physical and psychosocial wellbeing [1], associated with a high economic impact [2]. The assessment of functional recovery is essential for prognosis and designing

Andréane Richard-Denis

andreane.rdenis@gmail.com

1 Faculty of Medicine, Department of medicine, University of Montreal, Pavillon Roger-Gaudry, S-749, C.P. 6128, succ. Centre-ville, Montreal, QC H3C 3J7, Canada

2 Hôpital du Sacré-Cœur de Montréal, 5400 Gouin Boul. West, Montreal, QC H4J 1C5, Canada

3 Faculty of Medicine, Department of surgery, University of Montreal, Pavillon Roger-Gaudry, S-749, C.P. 6128, succ. Centreville, Montreal, QC H3C 3J7, Canada

4 Sainte-Justine University Hospital Research Center, 3175 Chemin de la Côte-Sainte-Catherine, Montréal, QC H3T 1C5, Canada a personalized rehabilitation program, and is therefore of central importance for patients and caregivers [3, 4].

Functional recovery after TSCI depends on the severity of the injury, age, and rehabilitation care $[5,6]$. When investigating chronic functional outcome after TSCI, some authors studied the functional status at 6 or 12 months after the injury [7-9]. Alternatively, others have included patients with a follow up of either 6 or 12 months interchangeably [10-12]. Although combining patients with either 6-month or 1-year functional assessment will maximize sample size for analyses, such methodology assumes that functional outcome remains similar during that period.

Hogel et al. [13] observed that the majority of individuals with tetraplegia and paraplegia had a significant increase in functional performance from 3 to 12 months after TSCI, regardless of the initial neurological status. However, they did study the pattern of improvement during this period, particularly after 6 months where the intensive functional rehabilitation (IFR) process is generally completed [14]. Wirth et al. [15] have investigated the functional recovery 1 , 
3, 6, and 12 months after TSCI. Unfortunately, motorincomplete injuries were not included in the analyses, which account for about $50 \%$ of all TSCI $[16,17]$. Results showed no significant change in the functional status from 6 to 12 months following motor-complete tetraplegia, while it increased significantly following motor-complete paraplegia [15]. However, the clinical significance of their results was not discussed based on existing criteria defined for identifying clinically significant improvement [18]. Another limitation of their study relates to the use of the second version of the Spinal Cord Independence Measure (SCIMII) that has been replaced by the third version (SCIM-III) including a new item for transfer ground-wheel chair and a modified scoring system for various sub-items [19].

Hence, the exact timing for reaching a chronic plateau during functional recovery remains unclear in patients sustaining a TSCI. Yet this information is important for setting realistic goals and expectations for patients, optimizing the use of resources and planning return to the community. In addition, the identification of patients with significant potential for functional improvement from 6 to 12 months post injury would also be very helpful to optimize their specific rehabilitation needs. Thus, the purpose of this study was twofold. The primary objective was to determine the extent of functional recovery from 6 to 12 months post TSCI, based on the neurological level of the injury. This will be accomplished by comparing the SCIM-III total score obtained at these two time points. The secondary objective was to identify patients achieving a significant clinical functional improvement (4 points or more in the SCIM total score) during this period, and determine which clinical characteristics are associated to this significant clinical improvement.

\section{Methods}

\section{Patients}

A prospective cohort of 125 patients sustaining a TSCI and admitted to a single level-I SCI-specialized trauma center between April 2010 and May 2016 was studied. Patients entered the cohort at the time of admission after consent and were followed at the same institution thereafter. They were included if they sustained an acute cervical (C1-C8) or thoraco-lumbar ( $\mathrm{T} 1$ and below) TSCI requiring surgical management in our institution and were aged 18 years or more. Patients were excluded if they sustained a penetrating trauma, received nonsurgical management or did not come to one or both follow-up visits. Since the purpose of the surgical management following TSCI is to decompress and stabilized the spine, it generally represents the standard of care for this population. Individuals that are not managed surgically following TSCI were thus excluded as they are generally to unstable to sustain spinal surgery and thus represent a distinct population. Similarly, individuals sustaining penetrating SCI were showed to present different characteristics and outcomes in comparison with individuals with blunt trauma [20]. The institutional review board approved this study and all patients were enrolled on a voluntary basis.

\section{Data collection}

Socio-demographic (age and sex), clinical, and trauma information was collected prospectively and updated on a daily basis during the acute care hospitalization. The initial severity of the TSCI was assessed upon arrival to the acute $\mathrm{SCI}$ center within $72 \mathrm{~h}$ of the injury and the initial American Spinal Injury Association impairment scale (AIS) grade from A to D was reported using the International Standards for Neurological Classification of SCI [21]. The neurological level of injury was stratified as tetraplegia (C1-C8) and paraplegia (T1-L2). The neurological status (AIS grade and neurological level of the injury, as well as the AIS motor score) was also assessed at the time of follow up at 6 and 12 months post injury.

Trauma severity (injury severity score-ISS) [22], velocity (high vs. low), as well as the presence of concomitant traumatic brain injury (TBI) were noted. The presence of TBI was noted by a trauma team specialized in TBI based on the Glasgow coma scale, level of consciousness, and presence of post-traumatic amnesia. The presence of spasticity during acute care was collected based on physical findings and symptoms reported by the patient, and required one of the following three criteria: (1) presence of increased velocity-dependant muscle tone at physical examination (Modified Ashworth scale score >1), (2) spasm and/or clonus noted at physical examination, and (3) spasm and/or clonus reported by the patient. Surgical delay was defined as the time (in hours) between the trauma and spinal surgery (time of skin incision), and was dichotomized as $<24 \mathrm{~h}$ vs. $\geq 24 \mathrm{~h}$. Acute care hospital length of stay was defined as the number of days from admission to discharge from the acute SCI center.

The occurrence of non-neurological complications during acute hospitalization was also determined. The most prevalent complications occurring after a TSCI [23] (pneumonia, pressure injuries, and urinary tract infections) were considered individually in the analyses. Pneumonia was diagnosed using clinical features and confirmed by a radiologist, using chest X-ray [24]. UTI were diagnosed using criteria from the 2006 Consortium for Spinal Cord Medicine Guidelines for healthcare provider [25], and pressure injuries were diagnosed using clinical guidelines defined by the National Pressure Ulcer Advisory Panel [26]. Complications 
occurring in less than $10 \%$ of patients (delirium, deep vein thrombosis, atelectasis, pulmonary embolism, acute respiratory distress syndrome, septicemia, etc.) were also included, but were considered altogether and referred as "other complications." The discharge destination from the acute care facility (IFR center vs. home with outpatient rehabilitation vs. transfer to referred hospital/nursing home) was also considered, as well as the length of stay in the IFR center for the concerned patients. Finally, the final destination for individuals who underwent IFR was also noted (discharge home vs. transitional rehabilitation facility (phase 3 rehabilitation) vs. nursing home/assisted group living). It is important to mention that in our public healthcare system, rehabilitation therapies are provided on an outpatient basis upon return home based on the evaluation of the treating multidisciplinary team in the acute care or IFR. Frequency and duration of the outpatient rehabilitation support is generally based on the needs and regional resources available, and was unfortunately not available in this study.

\section{Outcome assessment}

The SCIM-III scale was used to assess the functional status 6 and 12 months post TSCI. The SCIM-III is a valid and reliable scale specifically designed to assess the ability of SCI patients to perform basic activities of daily living [27, 28]. The SCIM-III assesses three domains: self-care (six items); respiration and sphincter management (four items); and mobility and transfers (nine items). The subscore for self-care ranges between 0 and 20, while respiration/ sphincter management and mobility/transfers subscores both range between 0 and 40 [27]. The total SCIM-III score can thus vary from 0 to 100 , with a higher score referring to a higher function. The difference in the SCIM-III total score between 6 and 12 months post-SCI was computed for each patient, and dichotomized as reaching or not a small clinical important difference, based on an improvement of 4 points proposed by Scivoletto et al. [18].

\section{Statistical analyses}

Continuous data were reported using mean ( \pm standard deviation), while categorical data were reported using percentage. The functional recovery was first assessed at a group level by comparing the mean SCIM-III scores obtained at 6 and 12 months post injury (Fig. 2). Then, the functional recovery was assessed individually and patients were classified based on whether they have reached or not a small clinical improvement ( $\geq 4$ points) in the total SCIM-III score during this interval of time. Mann-Whitney $U$ tests for continuous data and chi-square tests for categorical data were then used to compare socio-demographic and clinical characteristics between the two groups. Subanalyses were conducted based on the neurological level on injury (tetraplegia vs. paraplegia). The neurological status change between 6 and 12 months, namely the degree of completeness (AIS grade), neurological level of injury, and the AIS motor score were also compared for the two groups in order to better interpret the results. The percentage and characteristics of patients reaching a significant clinical improvement ( $\geq 10$ points) was also noted [18].

A total of 14 variables were considered as potential predictors of reaching a small clinically significant improvement in SCIM-III total score from 6 to 12 months post injury. The following variables were initially considered based on their potential influence of the functional recovery [9-12]: (1) age; (2) sex; (3) motor completeness of TSCI (complete AIS A/B vs. incomplete AIS C/D); (4) neurological level of injury (tetraplegia vs. paraplegia); (5) trauma velocity (high vs. low); (6) ISS; (7) occurrence of any complication during acute care; (8) surgical delay (<24 h vs. $\geq 24$ h); (9) presence of early spasticity; (10) LOS in acute care; (11) discharge destination (IFR center vs. others); (12) length of stay in IFR; (13) discharge orientation after IFR; (14) functional status 6 months; (15) AIS grade change from 6 to 12 months post injury; (16) AIS motor score change from 6 to 12 months post injury; and (17) change in the neurological level of injury between 6 to 12 months post injury. Univariate logistic regression analyses were first performed to select variables to be included in the multivariable regression model without exceeding the number of candidate predictors allowed for a cohort of 125 patients based on Green et al. [29]. Correlation analyses were also performed to exclude collinear variables $(r>0.7)$. A level of significance of 0.1 was used only at this step to avoid a type 2 error. Significant variables (age, motor completeness of the SCI, discharge in IFR, acute care, and IFR length of stay and functional status at 6 months post injury) were then included as candidate predictors in a multivariable logistic regression, using a backward elimination method to assess their potential influence on the studied outcome. The same process was also used in additional analyses to determine factors associated with the IFR length of stay (dependant variable) for individuals transferred to IFR after acute care $(N=92,73.6 \%$ of the final cohort), except that multivariable linear regression analyses were used (general lineal model). All statistical analyses were performed using the IBM SPSS Statistics 25 Software (Chicago, IL) and results were considered statistically significant when $p<0.05$.

\section{Results}

As showed in Fig. 1, a total of 271 patients fulfilled the inclusion criteria. A total of 146 patients did not show up at 
either 6 or 12 months follow-up visit and thus, 125 individuals with a mean age of $47.2 \pm 17.3$ years old were included in the analyses. Individuals included in the final cohort and individuals loss to follow up were similar in terms of age $(47.3 \pm 17.3$ vs. $56.6 \pm 20.9$ years old, respectively, $p=0.19)$, level of injury (53.6\% vs. $65.1 \%$ of tetraplegia respectively, $p=0.06)$, and AIS grade $(p=$ $0.25)$. The socio-demographic and clinical characteristics of individuals sustaining tetraplegia $(n=69,55.2 \%)$, paraplegia ( $n=56,44.8 \%)$, as well as for the entire cohort $(n=$ $125)$ are presented in Table 1 . Patients with tetraplegia were older, mainly sustained a motor-incomplete TSCI (AIS grade C or D), and a lower burden of associated traumatic injuries, which is consistent with the Canadian SCI population [17]. These individuals also developed medical complications (spasticity, pneumonia, UTI, and pressure injury) in a higher proportion; they were hospitalized in

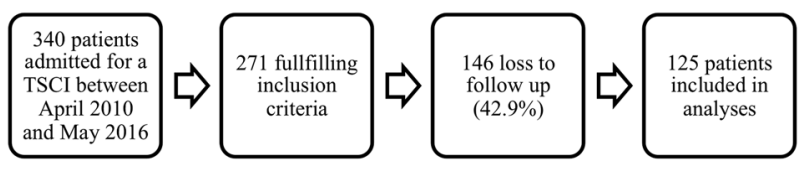

Fig. 1 Flow chart of patients included in the final cohort for analyses acute care longer and were less likely to be transferred to IFR than individuals with paraplegia (Table 1).

Figure 2 presents the SCIM-III total score between 6 and 12 months following the injury based on the neurological level of injury. The final cohort showed a statistically significant increase of 2 points in the SCIM-III total score between 6 and 12 months after TSCI. Within that group, individuals with tetraplegia improved significantly by 2 points between 6 and 12 months post TSCI, while the change in SCIM-III total score for individuals with paraplegia did not reach a statistical significance.

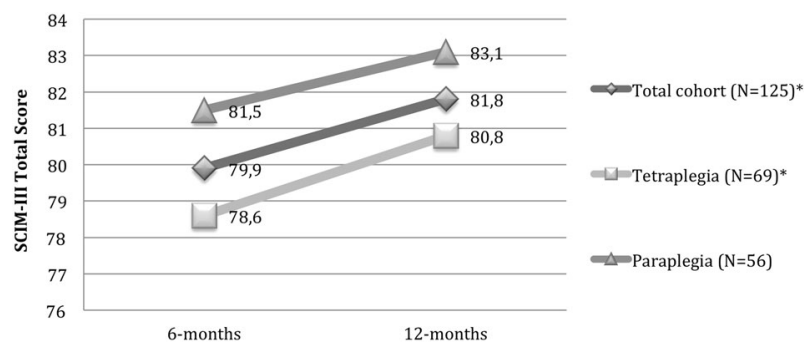

Fig. 2 SCIM-III total score between 6 and 12 months following a TSCI based on the initial neurological level of injury
Table 1 Baseline characteristics of the final cohort of individuals with TSCI $(N=125)$ and comparison for individuals with tetraplegia $(N=69)$ and paraplegia $(N=56)$

\begin{tabular}{|c|c|c|c|c|}
\hline & $\begin{array}{l}\text { Final cohort } \\
N=125\end{array}$ & $\begin{array}{l}\text { Tetraplegia } N=69 \\
(55.2 \%)\end{array}$ & $\begin{array}{l}\text { Paraplegia } N=56 \\
(44.8 \%)\end{array}$ & $p$-value \\
\hline Age $($ mean $\pm \mathrm{SD})$ & $47.2 \pm 17.3$ & $51.1 \pm 17.6$ & $42.5 \pm 15.7$ & $\mathbf{0 . 0 2}$ \\
\hline Sex (\% male $)$ & 80.0 & 81.2 & 78.6 & 0.81 \\
\hline SCI completeness ( $\%$ AIS A or B) & 39.2 & 29.0 & 51.8 & $\mathbf{0 . 0 1}$ \\
\hline ISS $($ mean \pm SD) & $22.7 \pm 9.6$ & $21.5 \pm 11.0$ & $24.2 \pm 7.2$ & $<10^{-3}$ \\
\hline Surgical delay $(\%<24 \mathrm{~h})$ & 42.4 & 33.3 & 53.6 & 0.01 \\
\hline Spasticity signs (\%) & 51.2 & 59.4 & 64.3 & $\mathbf{0 . 0 2}$ \\
\hline TBI $(\%)$ & 44.7 & 52.2 & 64.3 & 0.24 \\
\hline Trauma velocity (\% high) & 51.2 & 47.8 & 55.4 & 0.41 \\
\hline $\begin{array}{l}\text { Occurrence of any } \\
\text { complication }(\%)\end{array}$ & 55.2 & 50.7 & 37.5 & 0.17 \\
\hline •Pneumonia & 20.0 & 25.5 & 14.0 & 0.21 \\
\hline -Urinary tract infection & 14.4 & 16.4 & 18.6 & 0.79 \\
\hline -Pressure injury & 15.2 & 21.8 & 11.6 & 0.28 \\
\hline -Other complications & 31.2 & 29.1 & 46.5 & 0.09 \\
\hline $\begin{array}{l}\text { LOS in days in acute care (mean } \\
\pm \mathrm{SD} \text { ) }\end{array}$ & $25.2 \pm 18.9$ & $26.4 \pm 21.9$ & $23.4 \pm 14.3$ & $<10^{-3}$ \\
\hline \multicolumn{5}{|l|}{ Discharge destination after acute care } \\
\hline$\%$ IFR & 73.6 & 66.7 & 81.6 & $\mathbf{0 . 0 1}$ \\
\hline $\begin{array}{l}\% \text { return home with outpatient } \\
\text { rehabilitation }\end{array}$ & 18.9 & 24.6 & 12.2 & \\
\hline$\%$ referred hospital/nursing home & 5.6 & 7.1 & 4.1 & \\
\hline LOS in days in IFR (mean \pm SD) & $75.4 \pm 39.0$ & $81.0 \pm 47.2$ & $69.0 \pm 26.0$ & 0.06 \\
\hline
\end{tabular}

TSCI traumatic spinal cord injury, ISS Injury severity score, TBI traumatic brain injury, LOS length of stay, $I F R$ intensive functional rehabilitation

Bold values indicate statistical significance $p<0.05$ 
A total of 38 individuals $(30.4 \%)$ achieved a small clinical improvement from 6 to 12 months post TSCI (Table 2). Figure 3 shows the functional status for both groups. Individuals reaching small clinical improvement had lower functional status 6 months after the injury $\left(67.6 \pm 24.3\right.$ vs. $\left.85.3 \pm 22.4 ; p<10^{-3}\right)$. Their mean SCIMIII total score improved significantly by 10 points from 6 to 12 months, reaching a closer functional status compared with their counterparts but still statistically inferior (77.4 \pm 23.2 vs. $83.8 \pm 24.6, p=0.004$ ) (Fig. 3). Only 15 patients

Table 2 Comparison of the socio-demographic and clinical characteristics for patients reaching (Group 2) or not (Group 1) a small clinically important functional improvement on the SCIM-III between 6 and 12 months post TSCI $(N=125)$

\begin{tabular}{|c|c|c|c|c|}
\hline & $\begin{array}{l}\text { Group } 1 \text { (improvement of } \\
<4 \text { points) } N=87(69.6 \%)\end{array}$ & $\begin{array}{l}\text { Group } 2 \text { (improvement of } \\
\geq 4 \text { points) } N=38(30.4 \%)\end{array}$ & $p$-value & $\begin{array}{l}\text { Individuals reaching } \\
\geq 10 \text { points } N= \\
15(12 \%)\end{array}$ \\
\hline Age (mean (SD)) & $45.3 \pm 17.0$ & $51.7 \pm 17.3$ & 0.06 & $52.6 \pm 17.8$ \\
\hline (median (IQR)) & $45.0(30.0-60.0)$ & $57.5(37.0-65.3)$ & & $57.5(36.8-68.3)$ \\
\hline Sex $(\%$ male $)$ & 78.2 & 84.2 & 0.48 & 86.7 \\
\hline \multicolumn{5}{|l|}{ SCI completeness } \\
\hline$\%$ AIS A & 28.7 & 39.5 & 0.01 & 46.7 \\
\hline$\%$ AIS B & 3.4 & 13.2 & & 13.3 \\
\hline$\%$ AIS C & 13.8 & 23.7 & & 13.3 \\
\hline$\%$ AIS D & 54.0 & 23.7 & & 26.7 \\
\hline \multicolumn{5}{|l|}{ Neurological level of injury } \\
\hline$\% \mathrm{C} 0-\mathrm{C} 4$ & 24.7 & 21.2 & 0.57 & 33.3 \\
\hline$\% \mathrm{C} 5-\mathrm{C} 8$ & 27.4 & 36.4 & & 33.3 \\
\hline$\% \mathrm{~T} 1-\mathrm{T} 8$ & 9.6 & 3.0 & & 0 \\
\hline \% T9-L2 & 38.4 & 39.4 & & 33.3 \\
\hline ISS $($ mean \pm SD) & $22.2 \pm 8.7$ & $24.0 \pm 11.3$ & 0.81 & $27.3 \pm 15.9$ \\
\hline (median (IQR)) & $20.0(17.0-26.0)$ & $21.0(16.0-29.0)$ & & $25.5(16.8-29.0)$ \\
\hline Surgical delay $(\%<24 \mathrm{~h})$ & 41.4 & 44.7 & 0.84 & 40.0 \\
\hline Spasticity signs $(\%)$ & 44.8 & 57.9 & 0.24 & 80.0 \\
\hline TBI (\%) & 46.5 & 39.5 & 0.69 & 40.0 \\
\hline Trauma velocity ( $\%$ high) & 37.9 & 47.4 & 0.08 & 40.0 \\
\hline $\begin{array}{l}\text { Occurrence of any } \\
\text { complication }(\%)\end{array}$ & 55.2 & 55.3 & 1.00 & 53.3 \\
\hline •Pneumonia & 15.8 & 34.2 & $\mathbf{0 . 0 3}$ & 40.0 \\
\hline •UTI & 15.8 & 15.8 & 1.00 & 20.0 \\
\hline -Pressure injury & 15.8 & 18.4 & 0.79 & 33.3 \\
\hline -Other complications & 31.6 & 39.5 & 0.41 & 40.0 \\
\hline $\begin{array}{l}\text { LOS acute care (mean } \pm \\
\text { SD) }\end{array}$ & $27.0 \pm 16.3$ & $23.5 \pm 26.3$ & 0.04 & $17.4 \pm 10.6$ \\
\hline (median (IQR)) & $24.0(16.0-35.0)$ & $16.0(11.0-26.0)$ & & $16.5(8.5-23.0)$ \\
\hline \multicolumn{5}{|c|}{ Discharge destination after acute care } \\
\hline$\%$ IFR & 60.9 & 92.1 & 0.01 & 93.3 \\
\hline $\begin{array}{l}\% \text { return home with } \\
\text { outpatient rehabilitation }\end{array}$ & 27.6 & 2.6 & & 6.7 \\
\hline $\begin{array}{l}\% \text { referred hospital or } \\
\text { nursing home }\end{array}$ & 8.0 & 0 & & 0 \\
\hline LOS in IFR (mean $\pm \mathrm{SD})$ & $64.2 \pm 38.2$ & $86.0 \pm 38.9$ & 0.01 & $109.9 \pm 38.1$ \\
\hline (median (IQR)) & $58.0(31.0-89.0)$ & $86.0(58.0-125.0)$ & & $125.0(87.0-130.5)$ \\
\hline \multicolumn{5}{|c|}{ Discharge destination after IFR } \\
\hline$\%$ home & 79.2 & 68.6 & 0.31 & 46.7 \\
\hline $\begin{array}{l}\% \text { transitional } \\
\text { rehabilitation facility }\end{array}$ & 17.0 & 31.4 & & 46.7 \\
\hline$\%$ nursing home & 3.8 & 0 & & 0 \\
\hline$\%$ unknown & 0 & 0 & & 6.7 \\
\hline $\begin{array}{l}\text { SCIM total score at } \\
6 \text { months }(\text { mean } \pm \text { SD) }\end{array}$ & $85.3 \pm 22.3$ & $67.6 \pm 24.3$ & $<10^{-3}$ & $58.7 \pm 25.1$ \\
\hline (median (IQR)) & $98.0(71.0-100.0)$ & $71.5(57.3-85.3)$ & & $65.5(36.3-81.5)$ \\
\hline
\end{tabular}

$S C I M$ spinal cord independence measure, $S C I$ spinal cord injury, ISS injury severity score, $U T I$ urinary tract infection, $T B I$ traumatic brain injury, $L O S$ length of stay, IFR intensive functional rehabilitation

Bold values indicate statistical significance $p<0.05$ 
(12\% of the final cohort) reached a significant clinical improvement ( $\geq 10$ points) during this period. These individuals also showed a significant lower functional status at 6 months post injury compared with individuals not reaching a significant functional improvement during this period $\left(58.7 \pm 25.1\right.$ vs. $\left.85.3 \pm 22.4, p<10^{-3}\right)$ (Fig. 3$)$. These individuals then improve their functional status within the next 6 months to reach $73.9 \pm 25.1$ at 12 months post injury, which still consisted in a significant lower functional status as opposed to individuals from Group $1(p<0.05)$. Sociodemographic data for individuals reaching a significant clinical improvement are also showed in Table 1. These individuals were aged $52.6 \pm 17.8$ years old, sustained a motor-complete SCI in a high proportion (46.7\% of AIS A) and sustained a high burden of associated injuries (ISS of

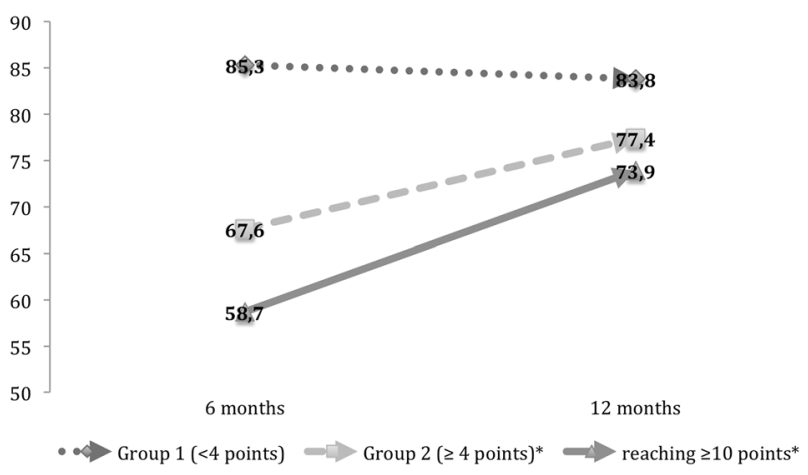

Fig. 3 SCIM-III total score between 6 and 12 months following TSCI for individuals reaching a small ( $\geq 4$ points) and a significant $(\geq 10$ points) clinical improvement, compared with individuals who did not reach functional improvement during this period
$26.2+/-15.6)$. In addition, a total of $80 \%$ of this subgroup developed sign or symptoms of spasticity during acute care.

As shown in Table 2, a higher proportion of motorcomplete TSCI (AIS grade A or B) was found in individuals reaching small clinical functional improvement. The two groups were otherwise similar in terms of sex, neurological level of injury, ISS, surgical delay, presence of spasticity, and TBI rate $(p>0.05)$ (Table 2$)$. There was a tendency toward older age and higher trauma velocity for Group 2 during this period (Table 2). Individuals from Group 2 were also hospitalized for a shorter time in acute care, were more likely to undergo IFR and were hospitalized around 20 days longer in IFR. This group also sustained a higher incidence of pneumonia during the acute care hospitalization compared with Group 1. On the other hand, individuals not reaching a small functional improvement between 6 and 12 months returned home directly after acute care in almost $30 \%$ compared with $2.6 \%$ in Group $1(p=0.01)$. The discharge orientation after IFR was similar for both groups, with around $75 \%$ returning home after IFR, around $25 \%$ transferred to a transitional rehabilitation facility and less than $3 \%$ to a nursing home after IFR.

Since the functional outcome is influenced by the neurological status, the neurological status change between 6 and 12 months post injury was also noted and compared for the two groups. As shown in Table 3, a total of $84.8 \%$ of the final cohort remained with the same degree of completeness of the injury (AIS grade) between 6 and 12 months post injury, with a similar proportion for Group 1 and 2 ( $p=$ 0.42 ). The conversion rate of the neurological level category (C1-C4, C5-C8, T1-T8, and T9 and below) as well as the
Table 3 Neurological status change between 6 and 12 months for patients reaching (Group 2) or not (Group 1) a small clinically important functional improvement on the SCIM-III between 6 and 12 months post TSCI $(N=125)$

\begin{tabular}{|c|c|c|c|c|}
\hline & $\begin{array}{l}\text { Entire } \\
\text { cohort } \\
N=125\end{array}$ & $\begin{array}{l}\text { Group } 1 \text { (improvement of } \\
<4 \text { points) } N=87(69.6 \%)\end{array}$ & $\begin{array}{l}\text { Group } 2 \text { (improvement of } \\
\geq 4 \text { points) } N=38(30.4 \%)\end{array}$ & $p$-value \\
\hline \multicolumn{5}{|c|}{ AIS grade conversion rate } \\
\hline$\%$ same grade & 84.8 & 82.1 & 91.2 & 0.42 \\
\hline $\begin{array}{l}\% 1 \text { grade } \\
\text { improvement }\end{array}$ & 11.6 & 14.1 & 5.9 & \\
\hline $\begin{array}{l}\% 2 \text { grades } \\
\text { improvement }\end{array}$ & 1.8 & 1.3 & 2.9 & \\
\hline$\%$ deterioration & 1,8 & 2.6 & 0 & \\
\hline \multicolumn{5}{|l|}{ NLI category change } \\
\hline$\%$ same level & 78.4 & 81.8 & 71.0 & 0.27 \\
\hline $\begin{array}{l}\% 1 \text { category } \\
\text { improvement }\end{array}$ & 12.4 & 7.6 & 22.6 & \\
\hline $\begin{array}{l}\% 2 \text { categories } \\
\text { improvement }\end{array}$ & 1.0 & 1.5 & 0 & \\
\hline$\%$ deterioration & 8.2 & 9.1 & 0 & \\
\hline $\begin{array}{l}\text { AIS motor score } \\
\text { change }(\text { mean }( \pm \mathrm{SD}))\end{array}$ & $1.6 \pm 3.9$ & $1.6 \pm 3.9$ & $1.5 \pm 4.2$ & 0.94 \\
\hline
\end{tabular}

AIS American Spinal Injury Association (ASIA) impairment scale, $N L I$ neurological level of injury, $S D$ standard deviation 
AIS motor score change during this period were also similar (Table 3).

The final multivariable logistic regression model showed that a longer hospitalization in IFR was the single factor significantly associated with reaching a small clinical functional improvement, with a small odd ratio $(\mathrm{OR}=1.01$ 95\% CI (1.00-1.03)). This model was statistically significant $\left(\mathrm{X}^{2}=5.9 ; p=0.02\right)$ for reaching a small clinically important improvement in SCIM-III total score from 6 to 12 months after the TSCI.

In order to better interpret the results, multivariable linear regression analyses (using a general linear model) were used to identify factors associated with the IFR length of stay. The final multivariable linear regression identifying factors associated with longer length of stay in IFR is shown in Table 4. A total of 88 patients (70.4\% of the final cohort) were transferred to a specialized IFR after acute care. Presence of early spasticity, longer surgical delay ( $>24 \mathrm{~h}$ ), higher burden of associated traumatic injury, and lower initial AIS motor score were significantly associated with increased IFR length of stay. This model was significant $(F=8.74, p=0.004)$ and explained $52.3 \%$ of the variance in the IFR length of stay.

\section{Discussion}

To our knowledge, this is the first study assessing the clinical and statistical significance of functional changes from subacute to chronic phase following TSCI. This study showed, from a prospective cohort study including 125 individuals, a statistically significant 2-point increase in the SCIM total score between 6 to 12 months after a TSCI. One third of cohort reached a small clinically significant functional improvement during this period. This finding highlights the importance of using an objective and validated criteria for defining significant clinical improvement when investigating the patterns of functional recovery in time. Although this difference was statistically significant, it did not reach a clinically significant level of 4 points. While this increase is in accordance with previous work $[13,15]$, this

Table 4 Factors associated with length of stay in intensive functional rehabilitation: results of the final general linear model $N=88$

\begin{tabular}{ll}
\hline Predictive factors & Beta coefficient $(95 \% \mathrm{CI})$ \\
\hline Absence of early spasticity & $-17.6(-31.3 ;-4.0)$ \\
Surgical delay Initial & $13.0(0.5 ; 25.4)$ \\
ISS & $1.1(0.4 ; 1.9)$ \\
Initial AIS motor score & $-0.7(-1.0 ;-0.5)$ \\
$R^{2}: 52.3 \%$ & \\
\hline
\end{tabular}

AIS grade American Spinal Injury Association (ASIA) impairment scale, LOS length of stay, ISS injury severity score study is the first to have accounted for the clinical significance of this result.

This study also showed that the functional improvement between 6 and 12 months was similar for individuals with tetraplegia and paraplegia (Fig. 2). A total of $30.4 \%$ of the cohort achieved a small clinical functional improvement, while only $12 \%$ of the cohort achieved a significant functional improvement during this period. This suggests that the functional status may reach a plateau before 12 months post injury. In other words, our results suggest that the functional recovery following TSCI tends to stabilize between 6 and 12 months post injury regardless of the neurological level of injury. This result is important knowing that individuals with tetraplegia in our cohort mainly sustained incomplete injuries, which is generally associated with higher neurological and functional outcomes when compared with complete injuries $[2,3]$. It may however explain why patients with tetraplegia showed a statistically significant improvement in functional recovery as opposed to patients with paraplegia. However, this difference was not found clinically meaningful.

Individuals who reached a small functional improvement between 6 and 12 months post injury (Group 2) showed a statistically lower functional status at 6 months compared with their counterparts. This may be explained by the fact that this group showed a higher severity of the TSCI, which is also associated with higher trauma velocity (Table 2). While this group improved their functional status from an average global SCIM score of 67-77 in the next 6 months (Fig. 3), Group 1 remained stable during this period. While this result may reflect a ceiling effect in Group 1 consisting of a high proportion of less severe injuries (AIS D), it also mostly highlights the importance of the distinction between the neurological and functional recovery in individuals with TSCI. Indeed, this study showed that individuals who reached a small clinical functional improvement between 6 and 12 months post injury were actually sustaining more severe TSCI.

While it is well recognized that individuals with high severity of SCI have poorer potential for neurological recovery, some of them will convert to motor-incomplete injuries [30] and/or will develop functional compensation, referring to changes in function independently of the neurological recovery [31]. Functional compensation consists in the use of assistive devices (wheelchairs, functional orthosis, computer technologies, etc.), adapted or new movement strategies, and/or the use of other procedures (such as tendon transfer surgery) aiming to reduce the handicap [31-33]. Our results showing that both groups sustained a similar neurological recovery during the studied period (Table 3) suggest that functional compensation (as opposed to neurological recovery) may be the main process of recovery in the subacute to chronic period following TSCI. Since the functional compensation consists in the main purpose of the IFR phase, 
our final multivariate logistic regression model further supports this result. This model showed that the IFR length of stay was the single factor significantly associated with reaching a small clinical functional improvement during this period. While the information on the rehabilitation therapy provided on an outpatient basis upon the return to community was not available in this study, these results may still reflect the importance of the specialized and coordinated rehabilitation resources provided throughout the continuum of care following TSCI.

The final linear multivariate regression model performed as an additional analysis to identify predictors of IFR length of stay showed that the development of early spasticity, higher surgical delay, higher burden of traumatic injuries, and lower initial AIS motor score as significant factors associated to longer IFR stay. These are few characteristics generally associated with more complex medical and rehabilitation management [11,34], which may translate into a longer rehabilitation phase. Results of this study may thus support rehabilitation efforts for more complex cases of TSCI as they may exhibit functional improvement in the subacute to chronic phase following TSCI.

It is also worth noting that the development of spasticity during acute care was associated to longer IFR length of stay, which is consistent with previous results [34]. Indeed, the development of early spasticity (during the acute care hospitalization following TSCI) was previously suggested to impede the IFR process and to increase the likelihood of transfer to inpatient transitional rehabilitation after IFR. However, how the presence of early spasticity impact the functional recovery process remains unclear in the literature. Indeed, while the spasticity following SCI was showed to be associated to pain, limited mobility, and reduced quality of life [35], this study also showed a growing proportion of early spasticity in individuals reaching a small and clinically significant functional improvement during the studied period. This finding may thus suggest that presence of early spasticity may also have a positive influence of the functional recovery process. However, future work should better study this clinical issue.

Individuals reaching a small clinical functional improvement between 6 and 12 months were more likely to undergo IFR and remained hospitalized longer both in acute and IFR than their counterparts. This result may again reflect the high severity of the injury for this group, requiring more complex medical care and being more vulnerable to medical complications such as pneumonia (Table 2), which was also shown to independently impact the long-term functional outcome [24, 36, 37]. Motorcomplete TSCI generally involves younger patients, which is in accordance with the previous literature and results of this study $[14,17]$. Because of the severity of their injuries and the complexity of their management, patients with severe TSCI should be managed in trauma centers and IFR facilities specialized in SCI care [38, 39]. Critical functional objectives should be achieved in this subgroup for safe return to the community, which may translates into longer IFR stay and explain results of this study.

Individuals reaching a small functional improvement between 6 and 12 months post injury were also significantly older than Group 1. This result is more likely related to the demographic characteristics of the aging TSCI population, frequently sustaining central cord syndrome, involving more severe impairment of the upper extremities [40]. Due to its motor-incomplete nature, it is generally associated with higher potential for neurological and functional recovery and may explain the strong tendency toward older age found in Group 2 [30].

\section{Limitations}

The main limitation of this study consists in the high rate of loss to follow up. Indeed, more than $40 \%$ of patients eligible did not present at either 6 or 12 months follow-up appointment. Due to their severe limitations in mobility and sphincter management, individuals with SCI generally present 20-30\% loss to follow-up rate [41]. The higher rate found in this study was most likely related to the fact that both follow up at 6 and 12 months was required to be included in the final cohort for analyses. However, this limitation may have not influenced results of this study since the baseline characteristics between individuals who were lost to follow up and those included in the final cohort were similar. It is also important to mention that the studied cohort was also similar to the Canadian SCI population [14].

Another limitation relates to the lack of information pertaining to the social environment and network, as well as functional status prior to the injury. These factors may influence function and return to community following TSCI, and should be considered in future studies. Finally, future studies may investigate the functional gain in the specific SCIM subscores in order to better understand and support functional improvement in the community setting during that period.

\section{Conclusions}

This prospective study among 125 TSCI patients showed that the functional status improved by a statistically significant 2 points in the SCIM-III total score from 6 to 12 months post injury. However, this functional improvement did not reach a clinical significant level of 4 points. Nevertheless, $30 \%$ of the final cohort did reach a small clinical functional improvement during this period. These individuals sustained more severe TSCI (AIS grade A or B) 
and showed a higher tendency toward older age and high trauma severity compared with their counterparts. While these results may be partly explained by the presence of a ceiling effect for individuals with less severe TSCI—such as the central cord syndrome generally associated with older age-, results of this study still show the potential for individuals with severe TSCI to significantly improve their functional status between 6 and 12 months post injury. Since the neurological recovery during this period was similar between the two groups, results may suggest that functional compensation is the main process underlying functional recovery in the subacute to the chronic phase following TSCI. Specialized and coordinated rehabilitation should be provided to support functional compensation throughout the continuum of care following TSCI. However, future work should investigate the impact of rehabilitation resources provided upon community reinsertion on the functional recovery during the subacute to chronic period, in order to adapt community resources accordingly.

\section{Data archiving}

Data sharing is not applicable to this article, as no datasets were generated or analyzed during the current study.

Funding This research was funded by the Fonds de Recherche Québec-Santé (Traumatology Research Consortium- AR-D), and by the US Department of Defense Spinal Cord Injury Research Program (JMM-T). Part of the data was collected through the Rick Hansen Spinal Cord Injury Registry.

Author contributions AR-D recruited the patients, participated to the design and management of this study, completed the analyses, interpreted the data, wrote the manuscript, and funded this study. RC participated to the recording of patients, to analyses, interpretation of the data, and participated to redaction of the manuscript. CT recorded and analyzed the data. JMM-T designed and managed the study, recruited the patients, interpreted the data, participated to redaction of the manuscript, and also funded this study.

\section{Compliance with ethical standards}

Conflict of interest The authors declare that they have no conflict of interest.

Ethic statement We certify that all applicable institutional and governmental regulations concerning the ethical use of human volunteers were followed during the course of this research.

Publisher's note: Springer Nature remains neutral with regard to jurisdictional claims in published maps and institutional affiliations.

\section{References}

1. Middendorp JHA, Donders A, Pouw M, Ditunno J, Curt A, Geurts A, et al. A clinical prediction rule for ambulation outcomes after traumatic spinal cord injury: a longitudinal cohort study. Lancet. 2011;377:1004-10.

2. Wilson JGR, Frankowski R, Kiss A, Davis A, Kulkarni A, Harrop $\mathrm{J}$, et al. A clinical prediction model for long-term functional outcome after traumatic spinal cord injury based on acute clinical and imaging factors. J Neurotrauma. 2012;29:2263-71.

3. Putz C, Akbar M, Grieser T, Wiedenho B, Furstenberg C, Gerner $\mathrm{H}$, et al. Neurological and functional recovery in multiple injured patients with paraplegia: outcome after 1 year. J Trauma Inj, Infect Crit Care. 2011;70:1078-85.

4. Whiteneck G, Gassaway J, Djikers MP, Heinemann AW, Kreider SE. Relationship of patient characteristics and rehabilitation services to outcomes following spinal cord injury: the SCIRehab Project. J Spinal Cord Med. 2012;35:484-502.

5. Al-Habib A, Ball J, Bajammal S, Casha S, Hurlbert J. Clinical predictors of recovery after blunt spinal cord trauma: systematic review. J Neurotrauma. 2009;28:1431-43.

6. Wilson JCD, Fehlings M. Clinical predictors of neurological outcome, functional status, and survival after traumatic spinal cord injury: a systematic review. J Neurosurg. 2012;17:11-26.

7. Facchinello Y, Beauséjour M, Richard-Denis A, Thompson C, Mac-Thiong JM. The use of regression tree analysis for predicting the functional outcome following traumatic spinal cord injury. J Neurotrauma. 2017. https://doi.org/10.1089/neu.2017. 5321. [Epub ahead of print].

8. Goulet J, Richard-Denis A, Mac-Thiong JM. Relationships between specific functional abilities and health-related quality of life in chronic spinal cord injury. Am J Phys Med Rehab. 2019;98:14-9.

9. Kaminski L, Cordemans V, Cernat E, M'Bra KI, Mac-Thiong JM. Functional outcome prediction after traumatic spinal cord injury based on acute clinical factors. J Neurotrauma. 2017;34:2027-33.

10. Richard-Denis A, Feldman D, Thompson C, Mac-Thiong JM. Prediction of functional recovery six months following traumatic spinal cord injury during acute care hospitalization. J Spinal Cord Med. 2018;41:309-17.

11. Richard-Denis A, Feldman DE, Thompson C, Mac-Thiong JM. Early predictors of global functional outcome after a traumatic spinal cord injury: a systematic review. J Neurotrauma. 2018;35:1705-25.

12. Wilson JR, Grossman RG, Frankowski RF, Kiss A, Davis AM, Kulkarni AV, et al. A clinical prediction model for long-term functional outcome after traumatic spinal cord injury based on acute clinical and imaging factors. J Neurotrauma. 2012;29:2263-71.

13. Hogel F, Mach O, Maier D. Functional outcome of patients 12 and 48 weeks after acute traumatic tetraplegia and paraplegia: data analysis from 2004-2009. Spinal Cord. 2012;50:517-20.

14. A look at the traumatic spinal cord injury in Canada: Rick Hansen Spinal Cord Registry (RHSCIR). J Spinal Cord Med. 2017;40:870-6. https://doi.org/10.1080/10790268.2017.1387124.

15. Wirth B, Van Hedel H, Kometer B, Dietz V, Curt A. Changes in activity after a complete spinal cord injury as measured by the spinal cord independence measure II (SCIM II). Neurorehabilit Neural Repair. 2008;22:146-53.

16. Dvorak MF, Noonan VK, Fallah N, Fisher CG, Rivers CS, Ahn H, et al. Minimizing errors in acute traumatic spinal cord injury trials by acknowledging the heterogeneity of spinal cord anatomy and injury severity: an observational Canadian cohort analysis. J Neurotrauma. 2014;31:1540-7.

17. Noonan VK, Kwon B, Soril L, Fehlings MG, Hurlbert RJ, Townson A, et al. The Rick Hansen Spinal Cord Injury Registry (RHSCIR): a national-patient registry. Spinal Cord. 2002;50:22-7.

18. Scivoletto GTF, Laurenza L, Molinari M. The spinal cord independence measure: how much change is clinically significant for spinal cord injury subjects. Disabil Rehabil. 2013;35:1808-13. 
19. Catz A, Itzkovich M, Tesio L, et al. A multicenter international study on the spinal cord independence measure, version III. Rasch psychometric validation. Spinal Cord. 2007;45:275-91.

20. Roach MJ, Chen Y, Kelly ML. Comparing blunt and penetrating trauma in spinal cord injury: analysis of a long-term functional and neurological outcomes. Top Spinal Cord Inj Rehabil. 2018;24:121-32.

21. Kirshblum SC, Burns SP, Biering-Sorensen F, Donovan W, Graves DE, Jha A, et al. International standards for neurological classification of spinal cord injury (revised 2011). J Spinal Cord Med. 2011;34:535-46.

22. Baker SP, O’Neill B. The injury severity score: an update. J Trauma. 1976;16:882-5.

23. Bourassa-Moreau E, Mac-Thiong JM, Ehrmann Feldman D, Thompson C, Parent S. Complications in acute phase hospitalization of traumatic spinal cord injury: does surgical timing matter? J Trauma Acute Care Surg. 2013;74:849-54.

24. Medicine CfSC. Respiratory management following spinal cord injury: a clinical practive guideline for health-care professionals. J Spinal cord Med. 2005;28:259-93.

25. Medicine CfSC. Bladder management for adults with adults with spinal cord injury: a clinical practive guideline for health-care providers. J Spinal cord Med. 2006;29:527-73.

26. NPUAP. NPUAP pressure ulcer stages/categories 2007. 2018. https://www.npuap.org/wp-content/uploads/2012/01/NPUAPPressure-Ulcer-Stages-Categories.pdf.

27. Catz A, Itzkovich M. Spinal cord independence measure: comprehensive ability rating scale for the spinal cord lesion patient. JRRD. 2007;44:65-8.

28. Bluvshtein V, Front L, Itzkovich M, Aidinoff E, Gelernter I, Hart $\mathrm{J}$, et al. SCIM III is reliable and valid in a separate analysis for traumatic spinal cord lesions. Spinal Cord. 2011;49:292-6.

29. Green SB. How many subjects does it take to do a regression analysis. Multivar Behav Res. 1991;26:499-510.

30. Fawcett JW, Curt A, Steeves JD, Coleman WP, Tuszynski MH, Lammertse D, et al. Guidelines for the conduct of clinical trials for spinal cord injury as developed by the ICCP panel: spontaneous recovery after spinal cord injury and statistical power needed for therapeutic clinical trials. Spinal Cord. 2007;45:190-205.
31. Curt A, Van Hedel HJ, Klaus D, Dietz V, EM-SCI Study Group. Recovery from a spinal cord injury: significance of compensation, neural plasticity, and repair. J Neurotrauma. 2008;25:677-85.

32. Kirshblum S, Millis S, McKinley W, Tulsky D. Late neurologic recovery after traumatic spinal cord injury. Arch Phy Med Rehabil. 2004;85:1811-7.

33. Dunn JA, Sinnott KA, Rothwell AG, Mohammed KD, Simcock JW. Tendon transfer surgery for people with tetraplegia: an overview. Arch Phys Med Rehabil. 2016;97:S75-80.

34. Richard-Denis A, Nguyen BH, Mac-Thiong JM. The impact of early spasticity on the intensive functional rehabilitation phase and community reintegration following traumatic spinal cord injury. $\mathrm{J}$ Spinal Cord Med. 2018:1-9. https://doi.org/10.1080/10790268. 2018.1535638. [Epub ahead of print].

35. Adams MM, Hicks AL. Spasticity after spinal cord injury. Spinal Cord. 2005;43:577-86.

36. Kopp MA, Watzlawick R, Martus P, et al. Long-term functional outcome in patients with acquired infections after acute spinal cord injury. Neurology. 2017;88:892-900.

37. Jaja B, Jiang F, Badhiwala JH, Schär R, Kurpad S, Grossman RG, et al. Association of pneumonia, wound infection and sepsis with clinical outcomes after acute traumatic spinal cord injury. J Neurotrauma. 2019. https://doi.org/10.1089/neu.2018.6245. [Epub ahead of print].

38. Richard-Denis A, Ehrmann Feldman D, Thompson C, BourassaMoreau É, Mac-Thiong JM. Cost and length of stay for the acute care of patients with motor-complete spinal cord injury following cervical trauma: the impact of early transfer to specialized acute SCI center. Am J Phys Med Rehabil. 2017;96:449-56.

39. Parent S, Barchi S, LeBreton M, Casha S, Fehlings M. The impact of specialized centers of care for spinal cord injury on length of stay, complications, and mortality: a systematic review of the literature. J Neurotrauma. 2011;28:1363-70.

40. Thompson C, Mutch J, Paren S, Mac-Thiong JM. The changing demographics of traumatic spinal cord injury: an 11-year study of 831 patients. J Spinal Cord Med. 2015;38:214-2.

41. Hwasoon K, Cutter G, George B, Chen Y. Understanding and preventing loss to follow-up: experiences from the Spinal Cord Injury Model Systems. Top Spinal Cord Inj Rehabil. 2018;24:97-109. 